\title{
Angioplastia Primária no Tratamento do Infarto Agudo do Miocárdio de Apresentação Noturna: "Suave é a Noite"?
}

Ver artigo relacionado na página 273

\author{
Luiz Alberto Mattos ${ }^{1}$
}

0 infarto agudo do miocárdio permanece a causa líder de mortalidade no Brasil, estimada em mais de 300 mil fatalidades anuais. ${ }^{1} \mathrm{O}$ advento da reperfusão coronária, quando aplicada até as primeiras 12 horas do início dos sintomas, reduziu os desfechos fatais, comprovando sua eficácia. ${ }^{2}$ Dessa forma, o esforço atual dos gestores de saúde tem sido direcionado para a disponibilização do tratamento em tempo hábil, seja por meio da lise farmacológica, com a aplicação dos fibrinolíticos, ou da lise mecânica, por meio da angioplastia transluminal coronária primária. ${ }^{3}$

Desde sua introdução por Hartzler et al. ${ }^{4}$, em 1983, a angioplastia transluminal coronária primária enfrentou uma saga de questionamentos e controvérsias, que demandou hercúlea convicção dos pioneiros do método. A constatação de resultados bastante consistentes ao longo do tempo levou a sua consolidação como tratamento preferencial no infarto agudo do miocárdio, atingindo a mais forte e unânime recomendação em todas as principais diretrizes (Classe I), com as múltiplas evidências de seu benefício (Nível A), quando aplicada, tempestivamente, por colegas aptos no manuseio dessa eficaz ferramenta terapêutica. ${ }^{3-6}$

A discussão sobre vantagens e desvantagens do tratamento foi salutar e trouxe benefícios inequívocos ao aperfeiçoamento do método intervencionista, levando a taxas de sucesso (restabelecimento do fluxo coronário anterógrado normal - TIMI 3) superiores a $80 \%$, redução das reoclusões aguda e subaguda do vaso-alvo, e durabilidade do resultado tanto clínico como angiográfico, reflexo da elevada permeabilidade tardia do vaso tratado, aspecto fundamental para preservação da função contrátil do ventrículo esquerdo e aumento significativo da sobrevida, quando comparado a outros métodos de reperfusão. ${ }^{7}$
A angioplastia transluminal coronária primária se consagrou por proporcionar reperfusão rápida, sustentada e eficaz, reduzidas taxas de reoclusão e reestenose, e preservação da microcirculação coronária, além de ser aplicada mediante pouca ou nenhuma contraindicação, sem o risco de complicações hemorrágicas graves. A evolução dos dispositivos (de balão para stents coronários, farmacológicos ou não) associada a terapia adjunta eficiente, baseada nos tienopiridínicos de segunda geração e nos inibidores de glicoproteína Ilb/IIla, fizeram toda a diferença ao longo de quase 30 anos. $^{8}$

Contudo, ao analisarmos as recomendações das diretrizes, seguem desafios a serem sobrepujados, como a aplicação do método 90 minutos após a confirmação clínica e eletrocardiográfica, por operadores experientes e em ambiente hospitalar apto a oferecer a infraestrutura necessária, isso tudo somado à demanda crescente para a transferência de pacientes provenientes de centros primários. . $^{3,5} 6$

Controvérsias atuais que envolvem a angioplastia transluminal coronária primária estão focadas em questões relacionadas ao momento mais adequado para o tratamento e na experiência acumulada do operador. Nesta edição, Cardoso et al. ${ }^{9}$ exploram uma dessas questões, qual seja, a possível diferença de resultados obtidos em infartados submetidos a angioplastia transluminal coronária primária realizada dentro e fora do horário de rotina. ${ }^{9}$

A noite será menos suave que o dia para a realização de angioplastia transluminal coronária primária? Parafraseando o romance escrito pelo ensaísta norte-americano F. Scott Fitzgerald, o tópico ainda merece considerações. A controvérsia se instala após a publicação de resultados de registro norte-america-

\footnotetext{
1 Serviço de Cardiologia Invasiva - Instituto Dante Pazzanese de Cardiologia - São Paulo, SP, Brasil. Correspondência: Luiz Alberto Mattos. Av. Dr. Dante Pazzanese, 500 - Vila Mariana - São Paulo, SP, Brasil - CEP 04012-909 E-mail: pivmattos@uol.com.br

Recebido em: 10/9/2010 • Aceito em: 12/9/2010
} 
no realizado por Magid et al. ${ }^{10}$, que concluem que a aplicação de reperfusão em pacientes com apresentação noturna ou nos finais de semana exibia maiores taxas de mortalidade, inclusive após angioplastia transluminal coronária primária. Contudo, após o ajuste de acordo com o retardo da aplicação da reperfusão (maior no atendimento noturno e nos finais de semana), a diferença deixava de ser significativa. Mas estava, assim, acesa mais uma polêmica acerca das múltiplas variáveis que influenciam os resultados desse método terapêutico. ${ }^{11}$

Afinal, por qual motivo a aplicação do mesmo método, nas mesmas instituições, levaria a resultados díspares, com maiores taxas de insucesso e óbito quando executados em horário noturno ou nos finais de semana? A resposta pode ser obtida com base em duas explicações, uma delas apoiada em evidências objetivas e outra dotada de subjetivismo, que nos remeterá às recomendações finais.

O ensaio randomizado CADILLAC, dedicado à comparação de estratégias intervencionistas no infarto agudo do miocárdio, demonstrou que aqueles tratados fora do horário de rotina (diurno, durante os dias da semana) apresentavam evidência de retardo um pouco maior para o tratamento (21 minutos; $\mathrm{P}<0,001$ ), porém sem impacto perceptível nas taxas de mortalidade imediatas e ao final de um ano (3,7\% vs. 4,7\%, diurno vs. noturno; $P=0,30) .{ }^{11}$ Mais robusta em seu conteúdo é a evidência proveniente do banco de dados da American Heart Association (Get With the Guidelines - Coronary Artery Disease), que incluiu 379 hospitais e 62.814 pacientes em cinco anos consecutivos. Nessa consistente pesquisa os autores concluíram não existir diferenças nas taxas de mortalidade hospitalar nos pacientes com infarto agudo do miocárdio submetidos a reperfusão, seja por meio de fibrinolíticos ou por angioplastia transluminal coronária primária $(12 \%$ e $62 \%$ dos tratamentos de reperfusão, respectivamente), tratados dentro e fora do horário de rotina das instituições. Contudo, a reperfusão realizada no horário noturno e nos finais de semana privou os pacientes de maior acesso à angioplastia transluminal coronária primária, e esta, quando realizada, mostrou retardo maior comparativamente ao horário de rotina dos atendimentos (25 minutos; $\mathrm{P}<0,001) .^{12}$

Cardoso et al. ${ }^{9}$ exploram essa controvérsia e em sua análise retrospectiva, unicêntrica e muito breve (dois meses de observação) chegam a conclusões semelhantes, em consonância com as evidências expostas previamente. A principal delas remete ao fato de o retardo entre os enfermos atendidos fora do horário de rotina ter sido significativamente superior ao daqueles reunidos no grupo oposto (43 minutos; $\mathrm{P}<0,001$ ). No entanto, a análise desse breve período de acompanhamento clínico, uma limitação da pesquisa, não apresenta a robustez suficiente para conclusões definitivas acerca do principal desfecho a ser observado, o óbito. A mortalidade foi maior que o dobro no atendimento noturno $(13,7 \%$ vs. $5,9 \%$; $P=0,28)$, levantando o questionamento de como se comportaria essa comparação, do ponto de vista estatístico, diante de uma amostra maior de pacientes. Outra diferença foi a tendência para maior prevalência de oclusão do tronco da coronária esquerda, como vaso-alvo, nos infartados reperfundidos fora do horário de rotina, cenário usualmente associado a desfechos catastróficos.

Assim, a possível diferença entre a realização da angioplastia transluminal coronária primária dentro e fora do horário de rotina converge para o fato de os pacientes atendidos fora do horário de rotina, comparativamente àqueles atendidos dentro do horário de rotina, apresentarem latência significativamente maior para a consumação da reperfusão mecânica (tempo porta-balão). Associa-se a essa constatação o conhecimento, já bem consolidado, de que quanto maior for esse retardo maiores serão as taxas de mortalidade, independentemente de os pacientes serem atendidos dentro ou fora do horário de rotina. Em análises prévias, na medida em que cresce o tempo despendido para obtenção da recanalização do vaso-alvo, a mortalidade se eleva significativamente, chegando a 50\%, quando se comparam atendimentos com latência inferior a 30 minutos a atendimentos com latência superior a 60 minutos. ${ }^{13}$ É muito provável que a análise de Cardoso et al. ${ }^{9}$ tenha sofrido o reflexo negativo dessa variável.

Outra explicação nos guia por uma via ainda pouco pavimentada por evidências e turva como a noite mais escura, composta de variáveis de origem múltipla e com a participação de diversos protagonistas. Nessa explicação inclui-se o conhecimento histórico de que nos hospitais, fora do horário de rotina, é frequente a atuação de profissionais de saúde mais jovens, menos experientes e menos afeitos à aplicação das diretrizes de forma estrita. Alguns registros demonstram que essas variáveis adversas existem de fato e não constituem apenas uma percepção cotidiana das rotinas dos hospitais, porém exploram muito timidamente a questão da experiência do cardiologista intervencionista atuante. ${ }^{14,15}$ É bem pouco provável que as instituições desejem explicitar os resultados comparativos de seus médicos com maior e menor experiência, considerando o possível efeito negativo dessa análise.

Em conclusão, a angioplastia transluminal coronária primária, quando efetivada tempestivamente, com retardo após a confirmação do infarto agudo do miocárdio de no máximo 120 minutos, por equipe de clínicos e intervencionistas aptos, seja na prescrição da correta farmacologia adjunta seja na aplicação das melhores técnicas intervencionistas (via de acesso, aspiradores, balões e stents coronários), apresentará resultados com potencial comprovado para reduzir as taxas de morbidade e mortalidade dessa síndrome, seja realizada durante o dia, à noite, em finais de semana ou feriados. 
A recomendação advinda da análise desses resultados é de que as instituições devem, cada vez mais, realizar análises temporais periódicas do desempenho das diversas demandas necessárias para o atendimento ótimo aos clientes infartados, observando tanto o retardo no atendimento em diversos momentos de decisão como seus responsáveis, fornecendo dados e atualizando protocolos e diretrizes de atendimento, e analisando criticamente os resultados de acordo com os operadores escalados, para que os pacientes recebam de fato, e em tempo hábil, o tratamento ótimo. ${ }^{14,15} \mathrm{O}$ atendimento aos pacientes na vigência de síndromes coronárias agudas deve ser multidisciplinar, dotado de liderança centralizada e respeitado, com interação plena entre socorristas, cardiologistas e intervencionistas, além de proporcionar suporte efetivo logístico da gestão hospitalar para que essas ações sejam de fato consumadas.

Só assim poderemos suspirar aliviados, com a certeza de que a noite será sempre suave, como na Riviera Francesa, tão bem retratada pelo reconhecido ensaísta norte-americano, perfeita não somente para os amantes apaixonados, protegidos pela luz das estrelas infinitas e pela magia da lua, mas para aqueles que sofrem a angústia e o medo decorrentes de infarto agudo do miocárdio em uma madrugada solitária.

\section{CONFLITO DE INTERESSES}

O autor declarou inexistência de conflito de interesses relacionado a este manuscrito.

\section{REFERÊNCIAS}

1. Brasil. Ministério da Saúde. DATASUS. Informações de Saúde. Epidemiologia e Morbidade [Internet]. [citado 2010 jul. 25]. Disponível em: http://www2.datasus.gov.br/DATASUS/index. php?area $=0203$

2. Fibrinolytic Therapy Trialists' (FTT) Collaborative Group. Indications for fibrinolytic therapy in suspected acute myocardial infarction: collaborative overview of early mortality and major morbidity results from all randomised trials of more than 1000 patients. Lancet. 1994;343(8893):311-22.

3. Mattos LA, Lemos Neto PA, Rassi Jr A, Marin-Neto JA, Sousa AGMR, Devito FS. Diretrizes da Sociedade Brasileira de Cardiologia - Intervenção Coronária Percutânea e Métodos Adjuntos Diagnósticos em Cardiologia Intervencionista (II Edição - 2008). Arq Bras Cardiol. 2008;91(6 Supl 1):1-58.

4. Hartzler GO, Rutherford BD, Mcconahay DR, Johnson WI Jr, McCallister BD, Gura GM Jr, et al. Percutaneous transluminal coronary angioplasty with and without thrombolytic therapy for treatment of acute myocardial infarction. Am Heart J. 1983;106(5 Pt 1):965-73.

5. Van de Werf F, Bax J, Betriu A, Blomstrom-Lundqvist C, Crea F, Falk $V$, et al. Management of acute myocardial infarction in patients presenting with persistent ST-segment elevation: the Task Force on the Management of ST-Segment Elevation Acute Myocardial Infarction of the European Society of Cardiology. Eur Heart J. 2008;29(23):2909-45.

6. Kushner FG, Hand M, Smith SC Jr, King SB 3rd, Anderson JL, Antman EM, et al. 2009 Focused Updates: ACC/AHA Guidelines for the Management of Patients With ST-Elevation Myocardial Infarction (updating the 2004 Guideline and 2007 Focused Update) and ACC/AHA/SCAI Guidelines on Percutaneous Coronary Intervention (updating the 2005 Guideline and 2007 Focused Update): a report of the American College of Cardiology Foundation/American Heart Association Task Force on Practice Guidelines. Circulation. 2009;120(22): 2271-306.

7. Huynh T, Perron S, O'Loughlin J, Joseph L, Labrecque M, TU JV, et al. Comparison of primary percutaneous coronary intervention and fibrinolytic therapy in ST-segment-elevation myocardial infarction: bayesian hierarchical meta-analyses of randomized controlled trials and observational studies. Circulation. 2009;119(24):3101-9.

8. Verheugt FW. Reperfusion therapy for ST-segment elevation myocardial infarction: trials, registries, and guidelines. Circulation. 2009;119(24):3047-9.

9. Cardoso CO, Quadros AS, Voltolini I, Azmus AD, Cardoso CR, Sebben J, et al. Angioplastia primária no infarto agudo do miocárdio: existe diferença de resultados entre as angioplastias realizadas dentro e fora do horário regular de trabalho? Rev Bras Cardiol Invasiva. 2010;18(3):273-80.

10. Magid DJ, Wang Y, Herrin J, McNamara RL, Bradley EH, Curtis JP, et al. Relationship between time of day, day of week, timeliness of reperfusion, and in-hospital mortality for patients with acute ST-segment elevation myocardial infarction. JAMA. 2005;294(7):803-12.

11. Sadeghi HM, Grines CL, Chandra HR, Mehran R, Fahy M, Cox DA, et al. Magnitude and impact of treatment delays on weeknights and weekends in patients undergoing primary angioplasty for acute myocardial infarction (the CADILLAC trial). Am J Cardiol. 2004;94(5):637-40.

12. Jneid $H$, Fonarow GC, Cannon CP, Palacios IF, Kilic T, Moukarbel GV, et al. Get With the Guidelines Steering Committee and Investigators. Impact of time of presentation on the care and outcomes of acute myocardial infarction. Circulation. 2008;117(19):2502-9.

13. Grines C, Patel A, Zijlstra F, Weaver WD, Granger C, Simes RJ. PCAT Collaborators. Percutaneous transluminal coronary angioplasty. Primary coronary angioplasty compared with intravenous thrombolytic therapy for acute myocardial infarction: six-month follow up and analysis of individual patient data from randomized trials. Am Heart J. 2003;145(1):47-57.

14. Pedersen SH, Galatius S, Hansen PR, Mogelvang R, Abildstrom $S Z$, Sørensen $R$, et al. Field triage reduces treatment delay and improves long-term clinical outcome in patients with acute ST-segment elevation myocardial infarction treated with primary percutaneous coronary intervention. J Am Coll Cardiol. 2009;54(24):2296-302.

15. Roger VL, Weston SA, Gerber Y, Killian JM, Dunlay SM, Jaffe $\mathrm{AS}$, et al. Trends in incidence, severity, and outcome of hospitalized myocardial infarction. Circulation. 2010;121(7):863-9. 